\title{
Visibility of the urethral meatus and risk of urinary tract infections in uncircumcised boys
}

\author{
Alexander Sasha Dubrovsky MDCM MSc, Bethany J. Foster MD MSCE, Roman Jednak MDCM, \\ Elise Mok PDt PhD, David McGillivray MD
}

Competing interests: None declared.

This article has been peer reviewed.

Correspondence to:

Alexander Sasha

Dubrovsky,

sasha.dubrovsky@mcgill.ca

CMAJ 2012. DOI:10.1503 /cmaj.111372

\begin{abstract}
Background: Uncircumcised boys are at higher risk for urinary tract infections than circumcised boys. Whether this risk varies with the visibility of the urethral meatus is not known. Our aim was to determine whether there is a hierarchy of risk among uncircumcised boys whose urethral meatuses are visible to differing degrees.
\end{abstract}

Methods: We conducted a prospective crosssectional study in one pediatric emergency department. We screened 440 circumcised and uncircumcised boys. Of these, 393 boys who were not toilet trained and for whom the treating physician had requested a catheter urine culture were included in our analysis. At the time of catheter insertion, a nurse characterized the visibility of the urethral meatus (phimosis) using a 3point scale (completely visible, partially visible or nonvisible). Our primary outcome was urinary tract infection, and our primary exposure variable was the degree of phimosis: completely visible versus partially or nonvisible urethral meatus.
Results: Cultures grew from urine samples from $30.0 \%$ of uncircumcised boys with a completely visible meatus, and from $23.8 \%$ of those with a partially or nonvisible meatus $(p=0.4)$. The unadjusted odds ratio (OR) for culture growth was 0.73 (95\% confidence interval $[\mathrm{Cl}] 0.35-1.52)$, and the adjusted OR was 0.41 (95\% Cl $0.17-0.95)$. Of the boys who were circumcised, $4.8 \%$ had urinary tract infections, which was significantly lower than the rate among uncircumcised boys with a completely visible urethral meatus (unadjusted OR 0.12 [95\% Cl 0.04-0.39], adjusted OR 0.07 [95\% Cl 0.02-0.26]).

Interpretation: We did not see variation in the risk of urinary tract infection with the visibility of the urethral meatus among uncircumcised boys. Compared with circumcised boys, we saw a higher risk of urinary tract infection in uncircumcised boys, irrespective of urethral visibility.
$\mathrm{U}$ rinary tract infections are one of the most common serious bacterial infections in young children. ${ }^{1-6}$ Prompt diagnosis is important, because children with urinary tract infection are at risk for bacteremia ${ }^{6}$ and renal scarring. ${ }^{1,7}$ Uncircumcised boys have a much higher risk of urinary tract infection than circumcised boys, ${ }^{13,4,4,68-12}$ likely as a result of heavier colonization under the foreskin with pathogenic bacteria, which leads to ascending infections. ${ }^{13,14}$ The American Academy of Pediatrics recently suggested that circumcision status be used to select which boys should be evaluated for urinary tract infection. ${ }^{1}$ However, whether all uncircumcised boys are at equal risk for infection, or whether the risk varies with the visibility of the urethral opening, is not known. It has been suggested that a subset of uncircumcised boys with a poorly visible urethral opening are at increased risk of urinary tract infection, ${ }^{15-17}$ leading some experts to consider giving children with tight foreskins topical cortisone or circumcision to prevent urinary tract infections. ${ }^{13,18-21}$

We designed a study to challenge the opinion that all uncircumcised boys are at increased risk for urinary tract infections. We hypothesized a hierarchy of risk among uncircumcised boys depending on the visibility of the urethral meatus, with those with a partially or nonvisible meatus at highest risk, and those with a completely visible meatus having a level of risk similar to that of boys who have been circumcised. Our primary aim was to compare the proportions of urinary tract infections among uncircumcised boys with a completely visible meatus with those with a partially or nonvisible meatus.

\section{Methods}

\section{Study design and participants}

We used a cross-sectional design conducted in a tertiary care pediatric emergency department that 
sees about 79000 patients per year. All boys aged 3 years or younger who were not toilet trained and for whom a catheter urine culture had been requested by their treating physician were eligible for inclusion in our study (most boys older than 3 years of age have obtained bladder control). ${ }^{22}$ Exclusion criteria included the use of antibiotic agents within the 72 hours preceding presentation to the emergency department, insertion of a catheter within the previous 7 days or congenital genitourinary anomalies that precluded the insertion of a catheter. Participants were divided into 3 groups: circumcised boys, uncircumcised boys with a partially or nonvisible urethral meatus and uncircumcised boys with a completely visible meatus.

We obtained urine samples for culture from boys presenting to the emergency department with signs or symptoms suggesting urinary tract infection. At our centre, children under the age of 3 months have a catheter urine sample obtained as a first-line test owing to the poor sensitivity of bag (nonsterile) urinalyses for urinary tract infection in this age group (sensitivity 0.77). ${ }^{23}$ At the discretion of the treating physician, samples from boys aged 3 months or older may undergo bag urinalysis (sensitivity 0.99$)^{23}$ before the decision is made to collect a urine sample via catheter. A positive urinalysis, defined as the presence of leukocyte esterase, nitrite and/or 10 or more white blood cells per high-powered field, ${ }^{1,23}$ triggers obtaining a sample via catheter.

We obtained written informed consent from the parents of the participants before the insertion of the catheter. Catheters were inserted, using a standard sterile technique, by nurses in the emergency department, who then completed a brief questionnaire. The Montreal Children's Hospital - McGill University Health Centre Research Ethics Board approved the study. This study is reported according to STROBE standards. ${ }^{24}$

\section{Outcome measures}

Our primary outcome measure was urinary tract infection, defined as growth of a single organism on culture $\left(\geq 10^{7}\right.$ colony-forming units $[\mathrm{CFU}] / \mathrm{L}) .{ }^{25}$ Our primary exposure variable was the degree of phimosis (i.e., the degree of visibility of the urethral meatus - completely visible v. partially or nonvisible). Because there are no validated scales of physiologic phimosis, we adapted those used in previous studies ${ }^{20,26}$ to create a simple 3-point scale to classify the degree of visibility of the urethral opening that best reflected what was seen during catheter insertion. At the time of insertion, the nurse assessed the degree of visibility by gently retracting the foreskin (avoiding any discomfort to the child) and comparing the observed anatomy to 3 drawings (Appendix 1, available at www.cmaj.ca /lookup/doi/10.1503/cmaj.111372). Interobserver reliability was determined by having a second nurse conduct an independent assessment on a convenience sample of the uncircumcised boys (63/309) just before their catheters were inserted. Only the assessment of the nurse inserting the catheter was used to classify urethral visibility in our primary analysis.

Technologists at the microbiology laboratory used a loop calibrated to deliver $0.001-\mathrm{mL}$ samples to inoculate blood and MacConkey agar plates. The plates were incubated at $35^{\circ} \mathrm{C}$ and examined daily for growth for 2 days. We considered plates that grew mixed cultures or fewer than $10^{7} \mathrm{CFU} / \mathrm{L}$ to be contaminated, and the organisms in them were not identified as per laboratory policy.

We collected data on the following potential confounders, all of which had been reported in previous studies as being risk factors for urinary tract infections in children: fever ${ }^{1,2,4,10,25}$ (temperature $>38^{\circ} \mathrm{C}$, either from parental report or as measured in the emergency department), previous urinary tract infections $\mathrm{s}^{1,2}$ (parental report), history of breastfeeding ${ }^{27}$ (parental report of having breastfed during the first 6 months of life), presence of bronchiolitis ${ }^{5}$ (treating physician's clinical diagnosis at time of visit) and age $\mathrm{e}^{10,28}$ ( $<3 \mathrm{mo}, \geq 3$ to $<12 \mathrm{mo}, \geq 12 \mathrm{mo}$ ). We collected data on visits to the emergency department from an electronic database.

\section{Sample size}

We estimated the size of the sample needed by comparing the rates of urinary tract infection between uncircumcised boys with completely visible versus partially or nonvisible meatuses. Previous studies in emergency departments reported rates of infection of up to $15 \%$ in circumcised boys and 35\% in uncircumcised boys. ${ }^{3,410,23}$ Assuming a hierarchy of risk for urinary tract infection among uncircumcised boys, the expected proportion of the group with completely visible meatuses with urinary tract infection was 15\% (approximating that of circumcised boys) versus 35\% for the group with partially visible or nonvisible meatuses. Using data from previous studies, ${ }^{16,26,29}$ we assumed the proportion of uncircumcised boys with a completely visible meatus would be $15 \%-25 \%$. We also assumed that an absolute difference of $20 \%$ in the rate of urinary tract infection between the groups with completely visible and partially or nonvisible meatuses would lead clinicians to consider these groups differently in management decisions. A sample of 320 uncircumcised boys would allow at least $80 \%$ power to detect a difference of 
$20 \%$ between groups using a 2 -sided $\chi^{2}$ test and an $\alpha$ level of 0.05 . We therefore planned to enrol a total of 400 boys, based on an estimated 20\% rate of circumcision in the local population..$^{23}$

\section{Statistical analysis}

We summarized patient characteristics as proportions for categorical variables and medians with interquartile ranges for continuous variables. We compared groups using the Mann-Whitney $U$ test for medians and $\chi^{2}$ test for proportions (or the Fisher exact test when the expected count was less than 5). We used unadjusted odds ratios (ORs) with 95\% confidence intervals (CIs) to compare the proportions of boys with urinary tract infections between groups. We conducted multivariable logistic regression following our unadjusted analyses, considering the following potential confounders: fever, previous urinary tract infection, history of breastfeeding, bronchiolitis and age. The final model included only those variables whose singular exclusion resulted in a change of $10 \%$ or more in the OR for the primary outcome.

To assess the impact of possible verification bias, we repeated our analysis using data from the subgroup of boys who had not undergone bag urinalysis before their catheter samples were taken. We assessed interobserver reliability of urethral visibility using $\mathrm{\kappa}$ statistics with $95 \%$ CIs, and we considered $p$ values of less than 0.05 to be statistically significant.

\section{Results}

We screened 440 patients for enrolment between April 2007 and December 2009. We enrolled 404 eligible patients (Figure 1). Urine culture results were available for 393 of these patients (97.3\%), who we included in our analysis. The 11 patients for whom urine cultures were missing were equally representative of the 3 groups. Our study population represents a convenience sample of $20.2 \%$ (393/1945) of all boys who provided a urine sample via catheter for culture in the emergency department during the study period. Of the 393 boys included in the analysis, 40 were uncircumcised with a completely visible meatus, 269 had a partially (106) or nonvisible (163) meatus, and 84 were circumcised. Interobserver reliability between the nurses' assessments of the visibility of the urethral meatus on the 3-point scale had a $\kappa$ statistic of $0.92(95 \%$ CI 0.84-1.01).

The characteristics of the patients are summarized in Table 1. The median age of the boys was

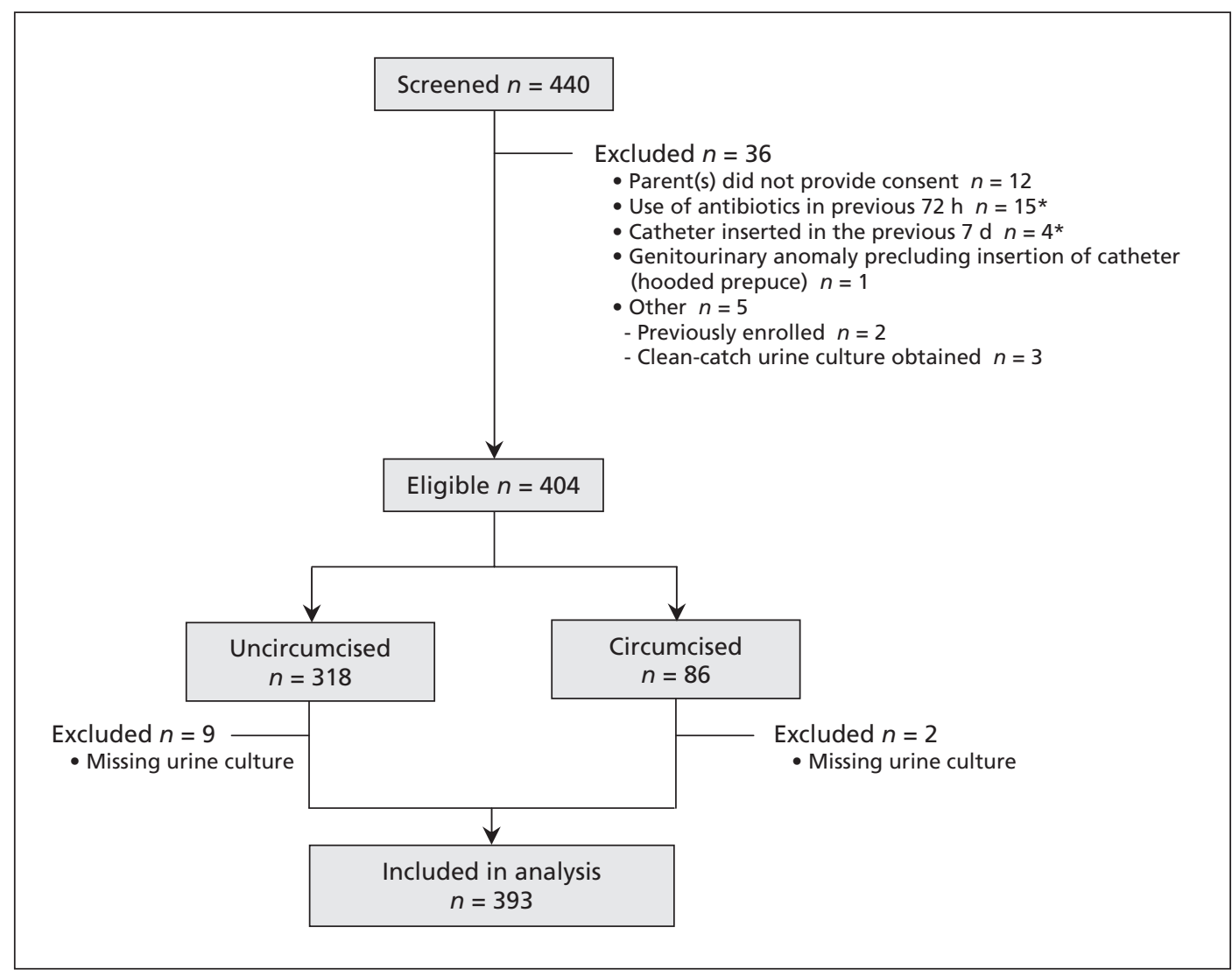

Figure 1: Selection of patients included in the study. *One patient met 2 exlusion criteria (use of antibiotics and previous insertion of a catheter). 
3.9 (interquartile range [IQR] 1.5-11.2) months; $44.0 \%$ (173/393) were younger than 3 months of age. Uncircumcised boys with a completely visible meatus were older (11.6 [IQR 2.8-18.5] mo) than those with a partially or nonvisible meatus (3.4 [IQR 1.4-8.9] mo). Only 7.9\% (11/140) of boys younger than 3 months had a completely visible meatus, compared with $32.3 \%$ (20/62) of boys 12 months of age or older.

Triage category, length of stay, time of presentation, visits that occurred on a weekend, triage complaints and diagnoses at discharge were similar among the 3 groups (Table 2). However, circumcised boys with a partially or nonvisible meatus were more frequently acutely triaged and were more likely to fall under the discharge diagnosis category of "rule out sepsis" than boys in the other groups. This finding is consistent with the younger age of this group (i.e., fever in infants under 3 months of age mandates triage category 2 at our institution). ${ }^{30}$ The rate of admission for boys with a partially or nonvisible meatus was $29.7 \%$ (80/269), compared with $15.0 \%$ (6/40) for boys with a completely visible meatus. However, the rate of admission after a positive urine culture showed no significant difference between these groups. In contrast, only $1.2 \%$ (1/84) of circumcised boys were admitted after a positive urine culture, compared with $8.4 \%$ (26/309) of uncircumcised boys.

Of the 393 boys included in the study, 80 $(20.4 \%)$ had urinary tract infections; the distribution of the uropathogenic organisms among the 3 groups of boys is shown in Table 3. Of boys with a completely visible meatus, $30.0 \%$ (12/40) had urinary tract infection, compared with $23.8 \%$ (64/269) of boys with a partially or nonvisible meatus $(p=0.4)$ (Table 4). The unadjusted OR for urinary tract infection in boys with a partially or nonvisible meatus versus those with a completely visible meatus was 0.73 (95\% CI 0.35 1.52) (Table 4). Age was the only variable we retained in our multivariable logistic regression. The adjusted OR was 0.41 (95\% CI $0.17-0.95 ; p$ $=0.04$ ), suggesting a significantly lower risk of urinary tract infection among boys with a partially or nonvisible meatus than among boys with a completely visible meatus.

To determine the impact of potential verification bias, we repeated our analysis with data from boys for whom a bag urinalysis had not been not done before the insertion of a catheter (97.0\% of boys aged $<3$ mo, $77.3 \%$ of boys aged $\geq 3$ mo but $<12$ mo, and $77.3 \%$ boys aged $\geq 12$ mo) (data not shown). The unadjusted OR for boys with a partially or nonvisible meatus versus a completely visible meatus was 1.17 (95\% CI 0.42-3.23); after adjusting for age, we calculated an OR of 0.55 (95\% CI $0.18-1.73$ ) (data not shown).

\section{Interpretation}

Previous studies have shown higher concentrations of periurethral uropathogenic bacteria in uncircumcised boys than in circumcised boys. ${ }^{13,14}$ After circumcision, the number of uropathogenic bacteria decreases substantially. ${ }^{19,21}$ The risk of uri-

Table 1: Characteristics of circumcised and uncircumcised boys enrolled in the study

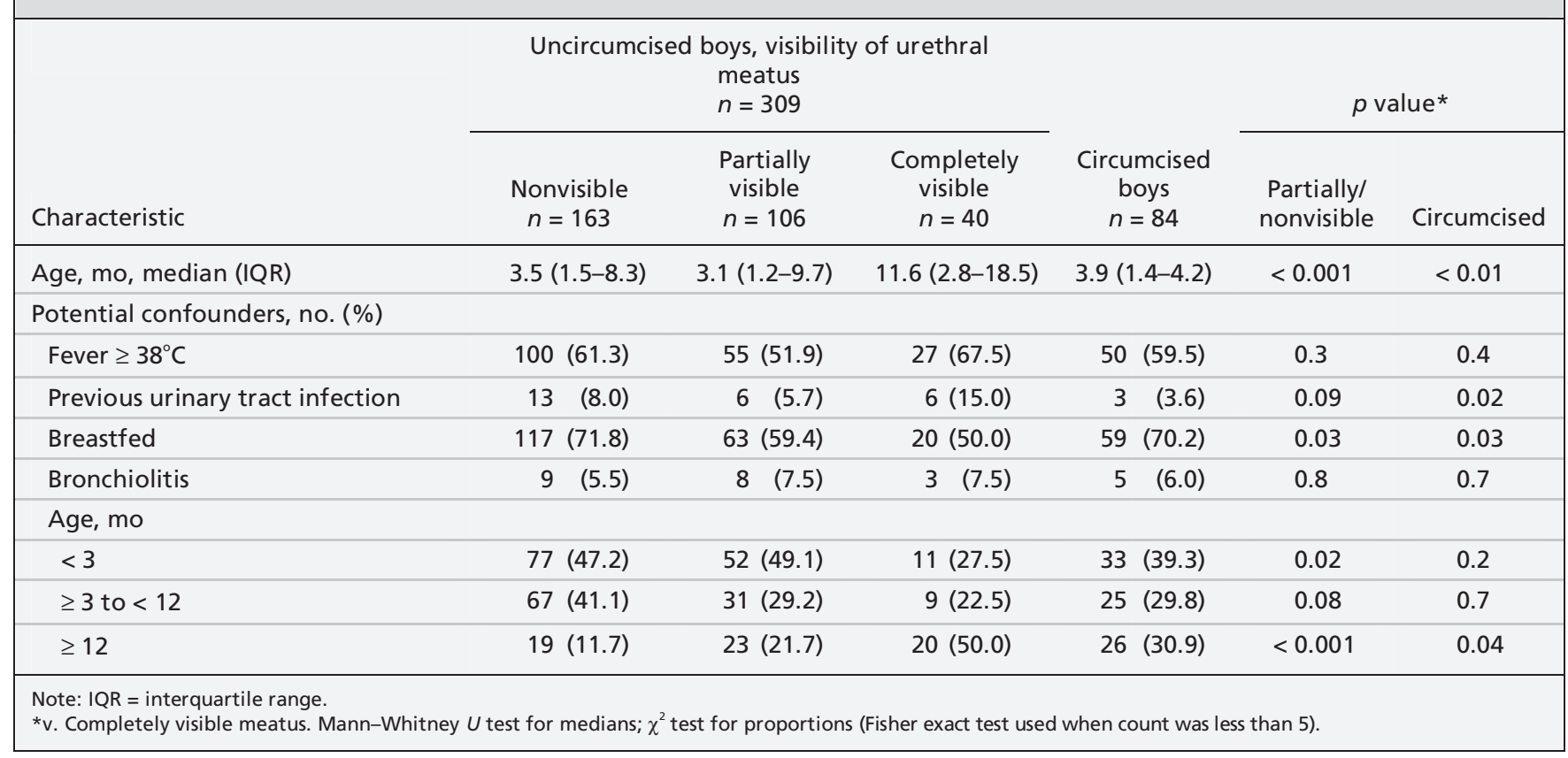


Table 2: Characteristics of presentations to the emergency department by participants of the study

\begin{tabular}{|c|c|c|c|c|c|}
\hline \multirow[b]{2}{*}{ Characteristic } & \multicolumn{2}{|c|}{$\begin{array}{l}\text { Uncircumcised boys, visibility } \\
\text { of urethral meatus, no. (\%) }\end{array}$} & \multirow[b]{2}{*}{$\begin{array}{c}\text { Circumcised } \\
\text { boys, no. (\%) } \\
n=84\end{array}$} & \multicolumn{2}{|c|}{$p$ value* } \\
\hline & $\begin{array}{c}\text { Partially or } \\
\text { nonvisible } \\
n=269\end{array}$ & $\begin{array}{c}\text { Completely } \\
\text { visible } \\
n=40\end{array}$ & & $\begin{array}{l}\text { Partially/ } \\
\text { nonvisible }\end{array}$ & Circumcised \\
\hline \multicolumn{6}{|l|}{ Triage category $\dagger$} \\
\hline $2(<15 \mathrm{~min})$ & $88(32.7)$ & $4(10.0)$ & $26(31.0)$ & 0.003 & 0.01 \\
\hline $3(15-30 \mathrm{~min})$ & $108(40.1)$ & $17(42.5)$ & $38(45.2)$ & 0.8 & 0.8 \\
\hline 4 (30-60 min) & $71(26.4)$ & $18(45.0)$ & $19(22.6)$ & 0.02 & 0.01 \\
\hline $5(60-120 \mathrm{~min})$ & $2(0.7)$ & $1 \quad(2.5)$ & $1 \quad(1.2)$ & 0.3 & 0.6 \\
\hline \multicolumn{6}{|l|}{ Length of stay, h‡ } \\
\hline$<4$ & $63(23.4)$ & $12(30.0)$ & $27(32.1)$ & 0.4 & 0.8 \\
\hline $4-8$ & $147(54.6)$ & $19(47.5)$ & $37(44.0)$ & 0.4 & 0.7 \\
\hline$\geq 8$ & 59 (21.9) & $9(22.5)$ & $20(23.8)$ & 0.9 & 0.9 \\
\hline \multicolumn{6}{|l|}{ Time of presentation $\ddagger$} \\
\hline Day (0800-1700) & $178(66.2)$ & $30(75.0)$ & $53(63.1)$ & 0.3 & 0.2 \\
\hline Evening (1700-0000) & $76(28.3)$ & $9(22.5)$ & $26(31.0)$ & 0.5 & 0.3 \\
\hline Night (0000-0800) & $15 \quad(5.6)$ & $1 \quad(2.5)$ & $5 \quad(6.0)$ & 0.4 & 0.4 \\
\hline Weekend & $30(11.2)$ & $10(25.0)$ & $14(16.7)$ & 0.02 & 0.3 \\
\hline \multicolumn{6}{|l|}{ Triage complaint§ } \\
\hline Fever & $138(51.3)$ & $18(45.0)$ & $38(45.2)$ & 0.5 & 0.9 \\
\hline Urinary symptoms & $17 \quad(6.3)$ & $5(12.5)$ & $9(10.7)$ & 0.2 & 0.8 \\
\hline Viral syndrome & $25 \quad(9.3)$ & $2 \quad(5.0)$ & $10(11.9)$ & 0.4 & 0.2 \\
\hline Vomiting & $20 \quad(7.4)$ & $4(10.0)$ & $10(11.9)$ & 0.6 & 0.7 \\
\hline $\begin{array}{l}\text { Vomiting and } \\
\text { diarrhea }\end{array}$ & $22 \quad(8.2)$ & $2 \quad(5.0)$ & $4 \quad(4.8)$ & 0.5 & 0.9 \\
\hline $\begin{array}{l}\text { Feeding } \\
\text { difficulty/dehydration }\end{array}$ & $9 \quad(3.3)$ & $0 \quad(0.0)$ & $2 \quad(2.4)$ & 0.2 & 0.3 \\
\hline Irritability & $8 \quad(3.0)$ & $0 \quad(0.0)$ & $(3.6)$ & 0.3 & 0.2 \\
\hline Referral for test & $2(0.7)$ & $1 \quad(2.5)$ & $1 \quad(1.2)$ & 0.3 & 0.6 \\
\hline Other & $28(10.4)$ & $8(20.0)$ & $7 \quad(8.3)$ & 0.08 & 0.06 \\
\hline \multicolumn{6}{|l|}{$\begin{array}{l}\text { Discharge diagnosis } \\
\text { category }\end{array}$} \\
\hline Fever without source & $39(14.5)$ & $6(15.0)$ & $16(19.0)$ & 0.9 & 0.6 \\
\hline Rule out sepsis & $37(13.8)$ & $0 \quad(0.0)$ & $9(10.7)$ & 0.01 & 0.03 \\
\hline Viral syndrome & $55(20.4)$ & $10(25.0)$ & $21(25.0)$ & 0.5 & 1 \\
\hline Genitourinary system & $46(17.1)$ & $13(32.5)$ & $6 \quad(7.1)$ & 0.02 & $<0.001$ \\
\hline Respiratory system & $14 \quad(5.2)$ & $2 \quad(5.0)$ & $3 \quad(3.6)$ & 0.9 & 0.7 \\
\hline $\begin{array}{l}\text { Gastrointestinal } \\
\text { system }\end{array}$ & $54(20.1)$ & $6(15.0)$ & $21(25.0)$ & 0.4 & 0.2 \\
\hline Other & $24 \quad(8.9)$ & $3 \quad(7.5)$ & $8 \quad(9.6)$ & 0.8 & 0.7 \\
\hline Admitted & $80(29.7)$ & $6(15.0)$ & $18(21.4)$ & 0.05 & 0.4 \\
\hline $\begin{array}{l}\text { Admitted with positive } \\
\text { urine culture }\end{array}$ & $21 \quad(7.8)$ & $5(12.5)$ & $1 \quad(1.2)$ & 0.3 & 0.006 \\
\hline \multicolumn{6}{|c|}{$\begin{array}{l}\text { *v. Completely visible meatus. } \chi^{2} \text { test (Fisher exact test used when count was less than 5). } \\
\text { tAs per the Canadian Triage and Acuity Scale. } \\
\text { tData on the time and day of presentation were collected from the emergency department database using the date and time the } \\
\text { patients were seen for triage. Length of stay defined as time between presentation to triage and discharge from the emergency } \\
\text { department. } \\
\text { §Created from complaints recorded in the emergency department database. "Urinary symptoms" included dysuria, frequency, } \\
\text { hematuria and penile complaints. "Viral syndrome" included upper and lower respiratory tract symptoms. "Referral for test" refers to } \\
\text { patients sent to the emergency department by their community physician for urine collection via catheter. "Other" included rash, } \\
\text { seizure, apnea and nonspecific complaints. } \\
\text { ICreated by merging similar physician diagnoses recorded in emergency department: viral syndrome (upper respiratory tract infection, } \\
\text { acute otitis media, croup), genitourinary system (urinary tract infection, dysuria, hematuria, urinary retention, balanitis), respiratory } \\
\text { system (bronchiolitis, pneumonia, respiratory distress), gastrointestinal system (gastroenteritis, vomiting, abdominal pain, feeding } \\
\text { problems, gastroesophageal reflux) and other (irritability, colic, apnea, apparent life-threatening event, febrile seizure, rash). }\end{array}$} \\
\hline
\end{tabular}


nary tract infection is about 10 times lower in circumcised boys than in uncircumcised boys, ${ }^{2,9-11,28}$ although the absolute risk difference is small. ${ }^{9,28}$ The reduced risk of urinary tract infection for circumcised boys that we found is similar to the reduced risks reported in previous studies. ${ }^{2,9-11,28}$

We thought that incomplete foreskin retractability with a poorly visible urethral meatus may be associated with increased risk of urinary tract infection. However, we found no difference in risk with degree of visibility of the urethral open- ing. Thus, we suggest that clinicians should consider circumcision status alone, not the degree of urethral visibility, when stratifying risk for boys presenting to the emergency department with symptoms or signs suggesting a urinary tract infection. This suggestion is in agreement with those of recently proposed clinical decision algorithms. ${ }^{1,11,12}$

After controlling for age, we found a significantly higher risk of urinary tract infection among boys with a completely visible meatus; this trend was seen across all age categories.

Table 3: Characteristics of participants' urine cultures

\begin{tabular}{|c|c|c|c|c|c|}
\hline \multirow[b]{3}{*}{ Contaminants $†$} & \multicolumn{2}{|c|}{$\begin{array}{l}\text { Visibility of urethral opening in } \\
\text { uncircumcised boys, no. (\%) }\end{array}$} & \multirow[b]{2}{*}{$\begin{array}{l}\text { Circumcised, no. } \\
\qquad \begin{array}{l}(\%) \\
n=84\end{array}\end{array}$} & \multicolumn{2}{|c|}{$p$ value* } \\
\hline & \multirow{2}{*}{$\begin{array}{c}\begin{array}{c}\text { Partially/non- } \\
\text { visible } \\
n=269\end{array} \\
48(17.8)\end{array}$} & $\begin{array}{l}\text { Completely } \\
\text { visible } \\
n=40\end{array}$ & & \multirow{2}{*}{$\begin{array}{c}\begin{array}{c}\text { Partially/ } \\
\text { nonvisible }\end{array} \\
0.004\end{array}$} & \multirow{2}{*}{ Circumcised } \\
\hline & & $0 \quad(0.0)$ & $(9.5)$ & & \\
\hline $\begin{array}{l}\text { Positive urine } \\
\text { culture }\end{array}$ & $64(23.8)$ & $12(30.0)$ & $4 \quad(4.8)$ & 0.4 & $<0.001$ \\
\hline Uropathogens & $n=64$ & $n=12$ & $n=4$ & & \\
\hline Escherichia coli & $41(64.1)$ & $9(75.0)$ & $4(100.0)$ & 0.2 & 0.003 \\
\hline Klebsiella species & $8(12.5)$ & $0 \quad(0.0)$ & $(0.0)$ & 0.3 & NA \\
\hline Proteus species & $3 \quad(4.7)$ & $3(25.0)$ & $(0.0)$ & 0.006 & 0.01 \\
\hline Other $¥$ & $12(18.8)$ & $0 \quad(0.0)$ & $(0.0)$ & 0.2 & NA \\
\hline \multicolumn{6}{|c|}{$\begin{array}{l}\text { Note: NA = not applicable. } \\
\left.{ }^{*} \text { V. Completely visible meatus. } \chi^{2} \text { test (Fisher exact test used when count was less than } 5\right) \text {. } \\
\text { tSamples grew mixed organisms and/or fewer than } 10^{7} \text { colony forming units per litre. } \\
\text { †Enterococcus (5), Pseudomonas (2), Enterobacter (1), Citrobacter (1), group B streptococci (1), Morganella (1) and Raoultella (1). }\end{array}$} \\
\hline
\end{tabular}

Table 4: Odds of urinary tract infection by circumcision status and by degree of visibility of the urethral meatus

\begin{tabular}{|c|c|c|c|c|}
\hline \multirow[b]{2}{*}{ Variable } & \multirow{2}{*}{$\begin{array}{c}\text { Participants, } \\
\text { no. }\end{array}$} & \multirow{2}{*}{$\begin{array}{l}\text { Positive urine } \\
\text { culture, no. (\%) }\end{array}$} & \multicolumn{2}{|c|}{ OR $(95 \% \mathrm{Cl})$} \\
\hline & & & Unadjusted & Adjusted* \\
\hline Uncircumcised boys & 309 & $76(25.0)$ & 1.00 & 1.00 \\
\hline Circumcised boys & 84 & $4 \quad(4.8)$ & $0.15(0.05-0.43)$ & $0.12(0.06-0.45)$ \\
\hline \multicolumn{5}{|l|}{ Uncircumcised boys } \\
\hline $\begin{array}{l}\text { Completely visible } \\
\text { urethral meatus } \\
\text { (reference) }\end{array}$ & 40 & $12(30.0)$ & 1.00 & 1.00 \\
\hline $\begin{array}{l}\text { Partially or nonvisible } \\
\text { urethral meatus }\end{array}$ & 269 & $64(23.8)$ & $0.73(0.35-1.52)$ & $0.41(0.17-0.95)$ \\
\hline Circumcised boys & 84 & $4 \quad(4.8)$ & $0.12(0.04-0.39)$ & $0.07(0.02-0.26)$ \\
\hline \multicolumn{5}{|l|}{ Age, mo } \\
\hline$<3$ (reference) & 173 & $30(17.3)$ & 1.00 & 1.00 \\
\hline$\geq 3$ to $<12$ & 132 & $45(34.1)$ & $2.47(1.45-4.20)$ & $2.71(1.53-4.80)$ \\
\hline$\geq 12$ & 88 & $5 \quad(5.7)$ & $0.29(0.11-0.77)$ & $0.27(0.09-0.77)$ \\
\hline
\end{tabular}


Although we cannot exclude the possibility that this group is truly at higher risk (contrary to our hypothesis), this result should be interpreted with caution given the small number of participants in the completely visible group. Furthermore, no previous studies suggest such an association, nor is there an apparent physiologic mechanism to explain this finding.

In addition, the higher OR for urinary tract infection among boys aged 3 months or older but less than 12 months, compared with those younger than 3 months of age, conflicts with the results of previous studies. ${ }^{9,11,28}$ The older boys in our cohort likely represented a higher-risk group, selected in some cases because of suspicious findings on bag urinalysis. Infants under the age of 3 months rarely underwent bag urinalysis before undergoing catheter insertion.

Finally, among older boys ( $\geq 12 \mathrm{mo}$ ), the risk of urinary tract infection was significantly lower than previously reported..$^{9,11,28}$

\section{Comparison with other studies}

Our results conflict with those of Hiraoka and colleagues,,$^{16}$ who found that boys with a tight foreskin had a 7.8-fold higher risk of febrile urinary tract infection than boys whose meatus was uncovered. That study involved 64 boys with febrile urinary tract infections and 714 healthy controls. The authors found that infants younger than 6 months with a diagnosis of febrile urinary tract infection were more likely to have a tightly covered urethral meatus than similarly aged healthy infants. However, their study was not designed to compare tight versus uncovered meatus and risk of urinary tract infection. Rather, they studied the foreskins of infants already given a diagnosis of febrile urinary tract infection and a reference group of healthy infants. However, the reference group referred to as healthy controls were not true controls, because they did not present with signs or symptoms of urinary tract infection, nor were urine samples obtained from them for culture. In addition, there was no attempt made to control for potential confounders, and only one observer examined the foreskin.

\section{Strengths and limitations}

We identified a well-defined cohort of boys presenting to the emergency department in whom urinary tract infection was suspected. In contrast to the study by Hiraoka and colleagues, ${ }^{16}$ we reduced susceptibility for selection bias by including boys with both positive and negative urine cultures from the same population of boys presenting to the emergency department. We also controlled for potential confounders, of which only age was retained in our model. In addition, multiple nurses assessed the visibility of the meatus, thereby reducing the risk of observer bias. Finally, we created an easy-to-use and reliable 3-point scale for determining the degree of visibility of the urethral opening; this scale may prove useful in future studies.

Owing to the relatively small number of boys with a completely visible meatus, significant differences were difficult to detect. Our study was powered to detect an absolute difference of $20 \%$ between 2 groups. It is possible that a difference smaller than $20 \%$ exists.

In addition, uncircumcised boys with a completely visible meatus were older than the boys in the other 2 groups. This trend likely reflects the natural history of physiologic phimosis, in which the foreskin becomes more retractable as boys age..$^{16,26,29}$

Finally, our cohort represents a highly select population for whom the treating physician requested a urine sample via catheter. Our analysis on the subgroup of boys for whom no urinalysis was done argues against potential verification bias. This result, coupled with the varied presentations of the children and their discharge diagnoses, suggests that our population is generalizable to all boys who are not toilet trained and who present to medical care with symptoms or signs suggesting urinary tract infection. Among these boys, the visibility of the meatus cannot be used to select those at risk for urinary tract infection.

\section{Conclusion}

Our results suggest that uncircumcised boys presenting with clinical symptoms or signs suggesting urinary tract infection are at equal risk for urinary tract infection irrespective of the visibility of the urethra. Clinicians should continue to use circumcision status alone, not the degree of phimosis, to decide which boys should undergo investigation for urinary tract infection.

\section{References}

1. Finnell SM, Carroll AE, Downs SM. Technical report — diagnosis and management of an initial UTI in febrile infants and young children. Pediatrics 2011;128:e749-70.

2. Craig JC, Williams GJ, Jones M, et al. The accuracy of clinical symptoms and signs for the diagnosis of serious bacterial infection in young febrile children: prospective cohort study of 15781 febrile illnesses. BMJ 2010;340:c1594.

3. Hsiao AL, Chen L, Baker MD. Incidence and predictors of serious bacterial infections among 57- to 180-day-old infants. Pediatrics 2006;117:1695-701.

4. Shaw KN, Gorelick M, McGowan KL, et al. Prevalence of urinary tract infection in febrile young children in the emergency department. Pediatrics 1998;102:e16.

5. Levine DA, Platt SL, Dayan PS, et al. Risk of serious bacterial infection in young febrile infants with respiratory syncytial virus infections. Pediatrics 2004;113:1728-34.

6. Ginsburg CM, McCracken GH Jr. Urinary tract infections in young infants. Pediatrics 1982;69:409-12.

7. Doganis D, Siafas K, Mavrikou M, et al. Does early treatment of urinary tract infection prevent renal damage? Pediatrics 2007; 120:e922-8. 
8. Singh-Grewal D, Macdessi J, Craig J. Circumcision for the prevention of urinary tract infection in boys: a systematic review of randomised trials and observational studies. Arch Dis Child 2005; 90:853-8.

9. To T, Agha M, Dick PT, et al. Cohort study on circumcision of newborn boys and subsequent risk of urinary-tract infection. Lancet 1998;352:1813-6.

10. Zorc JJ, Levine DA, Platt SL, et al. Clinical and demographic factors associated with urinary tract infection in young febrile infants. Pediatrics 2005;116:644-8.

11. Shaikh N, Morone NE, Bost JE, et al. Prevalence of urinary tract infection in childhood: a meta-analysis. Pediatr Infect Dis $J$ 2008; 27:302-8.

12. Newman TB, Bernzweig JA, Takayama JI, et al. Urine testing and urinary tract infections in febrile infants seen in office settings: the Pediatric Research in Office Settings' Febrile Infant Study. Arch Pediatr Adolesc Med 2002;156:44-54.

13. Wiswell TE, Miller GM, Gelston HM Jr, et al. Effect of circumcision status on periurethral bacterial flora during the first year of life. J Pediatr 1988;113:442-6.

14. Fussell EN, Kaack MB, Cherry R, et al. Adherence of bacteria to human foreskins. J Urol 1988;140:997-1001.

15. Tokgöz H, Polat F, Tan MO, et al. Preputial bacterial colonisation in preschool and primary school children. Int Urol Nephrol 2005;37:101-5

16. Hiraoka M, Tsukahara $\mathrm{H}$, Ohshima $\mathrm{Y}$, et al. Meatus tightly covered by the prepuce is associated with urinary infection. Pediatr Int 2002;44:658-62.

17. Shim YH, Lee JW, Lee SJ. The risk factors of recurrent urinary tract infection in infants with normal urinary systems. Pediatr Nephrol 2009;24:309-12.

18. Lee JW, Cho SJ, Park EA, et al. Topical hydrocortisone and physiotherapy for nonretractile physiologic phimosis in infants. Pediatr Nephrol 2006;21:1127-30.

19. Guünsar C, Kurutepe S, Alparslan O, et al. The effect of circumcision status on periurethral and glanular bacterial flora. Urol Int 2004; $72: 212-5$

20. Marques TC, Sampaio FJ, Favorito LA. Treatment of phimosis with topical steroids and foreskin anatomy. Int Braz J Urol 2005; 31:370-4.

21. Wijesinha SS, Atkins BL, Dudley NE, et al. Does circumcision alter the periurethral bacterial flora? Pediatr Surg Int 1998;13:146-8.

22. Oppel WC, Harper PA, Rider RV. The age of attaining bladder control. Pediatrics 1968;42:614-26.

23. McGillivray D, Mok E, Mulrooney E, et al. A head-to-head comparison: "clean-void" bag sersus catheter urinalysis in the diagnosis of urinary tract infection in young children. $J$ Pediatr 2005; 147:451-6.

24. von Elm E, Altman DG, Egger M, et al. The Strengthening the Reporting of Observational Studies in Epidemiology (STROBE) statement: guidelines for reporting observational studies. Lancet 2007;370:1453-7.

25. Urinary tract infection: clinical practice guideline for the diagnosis and management of the initial UTI in febrile infants and children 2 to 24 months. Pediatrics 2011;128:595-610.
26. Kayaba H, Tamura H, Kitajima S, et al. Analysis of shape and retractability of the prepuce in 603 Japanese boys. J Urol 1996; 156:1813-5

27. Ladomenou F, Moschandreas J, Kafatos A, et al. Protective effect of exclusive breastfeeding against infections during infancy: a prospective study. Arch Dis Child 2010;95:1004-8.

28. Schoen EJ, Colby CJ, Ray GT. Newborn circumcision decreases incidence and costs of urinary tract infections during the first year of life. Pediatrics 2000;105:789-93.

29. Gairdner D. The fate of the foreskin, a study of circumcision. BMJ 1949;2:1433-7.

30. Warren DW, Jarvis A, LeBlanc L, et al. Revisions to the Canadian Triage and Acuity Scale paediatric guidelines (PaedCTAS). CJEM 2008;10:224-43.

Affiliations: From the Divisions of Pediatric Emergency Medicine (Dubrovsky, McGillivray), Pediatric Nephrology (Foster), Pediatric Urology (Jednak), the Department of Epidemiology, Biostatistics and Occupational Health (Foster) and Montreal Children's Hospital Clinical Research Centre (Mok), Montréal Children's Hospital, McGill University Health Centre, Montréal, Que.

Contributors: Alexander Dubrovsky contributed to the conception and design of the study, the analysis and interpretation of the data, drafting the article and revising it for important intellectual content. Bethany Foster, Roman Jednak and David McGillivray contributed to the conception and design of the study and revised the manuscript for important intellectual content. Elise Mok contributed to the analysis and interpretation of the data, drafting the article and revising it for important intellectual content. All of the authors approved the final version of the manuscript submitted for publication.

Funding: This study received funding from the Montreal Children's Hospital Research Institute Clinical Projects Funding Competition for fellowship projects. The study sponsors had no role in the design of the study, the collection, analysis or interpretation of data, the writing of the report or the decision to submit the article for publication.

Acknowledgements: The authors thank Robert Rodrigues for his expert technical support and assistance in accessing the emergency department database, and Xun Zhang for his statistical expertise, support and critical review of the results. The authors also thank each of the emergency department nurses whose support and clinical expertise led to the success of this study. All of the authors are members of the Research Institute of the McGill University Health Centre, which is supported by the Fonds de Recherche du Québec - Santé. 